\title{
Neurocysticercosis: regression of a fourth ventricular cyst with praziquantel
}

\author{
David A Allcut, Alan Coulthard
}

\begin{abstract}
Symptomatic intraventricular cysticerci are said to respond poorly to the anticestodial agent praziquantel. $A$ case is reported of rapid regression of a large cyst of the fourth ventricle after oral praziquantel, avoiding the need for surgery.
\end{abstract}

Before 1979, treatment for neurocysticercosis involved excision of symptomatic cysts and shunting of obstructive hydrocephalus. ${ }^{1}$ More recently praziquantel has proved useful in parenchymal and basal/meningeal neurocysticercosis, where reduction in cyst size and often complete disappearance of cysts have been noted. ${ }^{23}$ In contrast, the response of intraventricular cysts to praziquantel therapy has been disappointing, leading to the recommendation that these cysts should be treated surgically and not with praziquantel. ${ }^{4}$

\section{Case report}

A 36 year old Asian female presented with a short history of intermittent diplopia and blurring of vision. She also complained of morning headache, nausea, vomiting and occasional unsteadiness of gait. There was no history of epilepsy. Her visual fields and acuity were normal but diplopia was present on upgaze and there was early bilateral papilloedema. A mild degree of disequilibrium was noted.

Computerised tomography showed multiple parenchymal calcifications and ventricular dilatation particularly involving the fourth ventricle (fig a). Subsequent immunofluorescent antibody testing was strongly positive for cysticercosis.

Insertion of a ventriculo-peritoneal shunt resulted in complete resolution of her headache, but the nausea, diplopia and gait disturbance persisted.

CT ventriculography via the shunt reservoir showed a large $(2.5 \times 1.5 \times 2 \mathrm{~cm})$ cyst within an enlarged fourth ventricle (fig b).

The patient declined surgical intervention, and so oral praziquantel therapy was initiated at a dose of $50 \mathrm{mg} / \mathrm{kg} /$ day, with prednisolone $40 \mathrm{mg} /$ day reducing to $30 \mathrm{mg} /$ day after three days. No adverse effects were noted.

Repeat CT ventriculogram after seven days of treatment showed a considerable reduction in the size of the cyst (fig c) and a repeat study after 21 days confirmed that the size reduction had been maintained. Treatment was discontinued after thirty days.

The symptoms of diplopia, nausea and unsteadiness improved considerably and at follow up one year after completing treatment the patient was asymptomatic.

\section{Discussion}

Surgical excision is currently the treatment of choice for symptomatic cysticerci within the fourth ventricle, ${ }^{5}$ despite significant risk of mortality and morbidity. ${ }^{67}$ There were $14.8 \%$ of patients who were left disabled in one


Figure a) Admission CT showing greatly enlarged fourth ventricle. Two calcified cysticerci are shown in the right frontal lobe. b) CT ventriculogram pre-treatment confirms a large cystic lesion within the enlarged fourth ventricle. $c$ ) Repeat $C T$ ventriculogram one week after starting praziquantel treatment. The cyst now measures less than one centrimetre and site of attachment to the ventricular wall is seen. 
series $^{6}$ and $26.3 \%$ died in another series. ${ }^{7}$ The poor results were largely attributed to secondary inflammatory changes leading to difficult dissection.

In comparison, treatment with praziquantel is safe, although an inflammatory response similar to the Jarisch Herxheimer reaction has been described. ${ }^{8}$ Simultaneous administration of corticosteroids prevents or attenuates this reaction. ${ }^{9}$

Our findings of rapid clinical and radiological response to oral praziquantel contrast with the findings of Vasconcelos $e t a l$ who reported no change in the appearance of fourth ventricular cysts in their series of four patients receiving oral praziquantel $50 \mathrm{mg} / \mathrm{kg} /$ day without steroid cover. Our patient received prednisolone in addition to praziquantel. Steroids alone do not appear to have any effect on the size of cysticercosis cysts. ${ }^{9}$

Our experience suggests that a trial of oral praziquantel is worthwhile in neurocysticercosis even for intraventricular cysts, in the hope of avoiding the morbidity and mortality associated with posterior fossa surgery.

We thank Dr J B Foster, Mr P J Crawford and Dr V L McAllister for allowing us to report this case.

1 Botero D, Castano S. Treatment of cysticercosis with praziquantel in Colombia. Am J Trop Med Hyg

2 Sotelo J, Escobedo F, Rodriguez-Carbajal J, Torres B, Rabbio-Donnadieu F. Therapy of parenchymal brain cysticercosis with praziquantel. N Eng J Med 1984;310:1001-7.

3 Robles C, Sedano AM, Vargas-Tentorini N, GalindoVirgew S. Long term results of praziquantel therapy in neurocysticercosis. J Neurosurgery 1987;66:359-63.

4 Vasconcelos $\mathrm{D}$, Cruz-Seguva $\mathrm{H}$, Mateas-Gomez H, Alanis GZ. Selective indications for the use of praziquantel in the treatment of brain cysticercosis. I Neurol Neurosurg Psychiatry 1987;50:383-88.

5 Apuzzo MLZ, Dobkin WR, Zee C, Chan JC, Gianotta SL Weiss MH. Surgical considerations in treatment of intraventricular cysticercosis. J Neurosurg 1984;60:400-7.

6 Loya M, Kleniga E, Estaval B. Fourth ventricular cysticerosis. Neurosurgery 1980;7:456-8.

7 Colli BO, Martelli N, Assivati JA, Machado HR, Forjaz SV. Results of surgical treatment of neurocysticercosis in 69
. cases. J Neurosurgery 1986;65:309-15.

8 Scully RD, Mark EJ, McNeely BU (Eds). Case records of the Massachusetts General Hospital. Case 11-1986: Cerebral cysticercosis. N Eng J Med 1986;314:767-74.

9 de Ghetaldi LD, Norman RM, Dockville AW. Cerebra cysticercosis treated biphasically with dexamethasone and praziquantel. Ann Intern Med 1983;99:179-81. 